Mini Review

\title{
Ectoparasitic viral zoonoses in the southern african context
}

\begin{abstract}
Climate changes can affect vector-borne diseases in the human population and can have an impact on viral pathogenicity in the host. There is an abundance of vector-borne diseases globally and a significant percentage is viral vector-borne diseases. Arboviruses are a group of viruses that are transmitted by mosquitoes, ticks or other arthropods. Some arboviral infections can present with clinical signs and symptoms of haemorrhagic fever and the classic example is Crimean-Congo haemorrhagic fever (CCHF). The epidemiological approach to a communicable disease involves the infectious agent, the mode of transmission and the host and CCHF fits this approach as it is transmitted by the Ixodid tick, Hyalomma sp and it can infect humans and other living animals. CCHF is the most important arboviral disease with haemorrhagic manifestations in Southern Africa. CCHF virus (CCHFV) has a negative sense single stranded genome with three segments, namely large (L), middle (M) and small (S). A recent research study in South Africa revealed high frequency reassortment in the $\mathrm{M}$ segment of CCHFV and further studies are indicated as to whether this is an advantage for increased transmissibility or enhanced pathogenicity. CCHV appears to be one of the most genetically diverse of the arbovirus grouping. There are no vaccine prophylactic and effective therapeutic interventions available for $\mathrm{CCHF}$ at this time and vector control is the mainstay intervention strategy available. CCHF is a notifiable disease in Southern Africa. Further research studies could improve intervention strategies for CCHF and possibly other arboviral diseases and could enhance outbreak control measures and surveillance strategies.
\end{abstract}

Keywords: Ectoparasite, Arthropod-borne, Arboviruses, Viral zoonosis, Southern Africa, Crimean Congo haemorrhagic fever, Communicable diseases
Volume 2 Issue I - 2015

\author{
Webber LM \\ Department of Medical Virology, University of Pretoria, South \\ Africa
}

Correspondence: LM Webber, Professor, Department of Medical Virology, University of Pretoria, Gauteng Province, Private Bag X323, Arcadia, Pretoria, 0007, South Africa, Tel +27I2 319 2656, Fax+27I2 3255550 Email Lynne.webber@up.ac.za

Received: November II, 2014 | Published: January 02, 2015
Abbreviations: VHF, Viral Haemorrhagic Fever; CCHF, Crimean Congo Haemorrhagic Fever; CCHFV, Crimean Congo Haemorrhagic Fever Virus; RFV, Rift Valley Fever; RNA, Ribonucleic Acid; L, Genomic Large Segment; M, Genomic Middle Segment; S, Genomic Small Segment

\section{Introduction}

Climate change can affect vector-borne diseases in the human population and can have a significant impact on viral pathogens in their host. Climate changes require enhancement of current surveillance systems and the development of effective global and local strategies to prevent vector-borne morbidity and mortality. ${ }^{1}$ In addition, there are many other factors that influence emergence or re-emergence of infectious diseases and where to look for these diseases is essential for designing and maintaining surveillance strategies. Two hot spot global regions have been identified, namely Southeast Asia where humans and animals coexist intimately and Sub-Saharan Africa that has a high disease burden and poor publichealth infrastructure. ${ }^{2}$ There is an abundance of vector-borne diseases globally and a significant percentage is viral vector-borne diseases. ${ }^{3}$ These viral diseases are examples of communicable diseases. ${ }^{4}$ Insectborne diseases have adopted a specific mode of transmission that makes many of these insect-borne diseases dependent on vectors for transfer or carriage from the reservoir to the new host. The mosquito is an important vector, as it is globally abundant, lives in very close proximity to human and needs to feed on blood. ${ }^{5}$ Mosquito-borne viral infections are of considerable public health interest especially due to the following concerns:

a) Potential epidemics.

b) Potentially fatal, untreatable diseases with significant morbidities and mortalities. c) And risks to health care personnel of contracting diseases after exposure to these infected patients. ${ }^{6,7}$

In contrast, ectoparasite zoonoses are a group of infections that are either vector-borne by ectoparasites, as zoonoses or as both. Humans become involved in the ectoparasitic cycle that feeds on blood. This is often accidental for the humans and may give rise to infection. A classic example of an ectoparasite zoonosis is plague which is maintained by flea vectors infecting rodents. Ticks are also ectoparasite vectors that can transmit diseases to humans. Ticks comprise two large families, the Ixodidae, or hard ticks, and the Argasidae, or soft ticks, hard ticks are responsible for the transmission of rickettsiae and arboviruses. Hard ticks can also be responsible for spreading arboviruses normally transmitted by mosquitoes, such as Japanese encephalitis, St Louis encephalitis, Eastern and Western equine encephalitis. ${ }^{8}$ Climate also plays a role in the life cycle of ticks as it can be modified by temperature and humidity. ${ }^{1}$

Arboviruses are a group of viruses that are transmitted to humans by mosquitoes, ticks or other arthropods. The first two letters of arthropod and borne make up the "arbo" that now designates this group of viruses as arthropod-borne. More than 100 arboviral diseases have been recognised worldwide. ${ }^{9}$ Some arbovirus infections can also present with clinical signs and symptoms of haemorrhagic fever and are then called viral haemorrhagic fevers (VHFs). A classic example of an arboviral disease with haemorrhagic fever is Crimean-Congo haemorrhagic fever (CCHF). CCHF is found in large regions in Africa, such as West, Central, East and South Africa, as well as Asia and is transmitted by the hard Hyalomma ticks. ${ }^{10} \mathrm{CCHF}$ is the most important viral haemorrhagic fever infection and disease in South Africa and a small number of cases are diagnosed annually. A larger number of suspected cases are investigated as part of the differential clinical diagnosis. ${ }^{11}$ The first indigenous case of CCHF was reported in 1981 but remains uncommon amongst humans although there 
is widespread evidence of it in animals throughout much of South Africa. ${ }^{12,13}$ A recent research study from South Africa has shown promising data of a high frequency of reassortment in a selected genetic cluster of this viral member of the Bunyaviridae family that may relate particularly to its pathogenicity and transmissibility. ${ }^{14}$

The purpose of this article is to review and highlight the following namely:

a) To review the disease role of viral vector-borne infections and revise it some terms frequently used in communicable disease epidemiology.

b) To provide a short background of arboviral diseases occurring in Southern Africa.

c) To describe ectoparasite zoonoses with tick-borne viral diseases as examples.

d) To discuss CCHF as the most important arboviral disease with haemorrhagic manifestations in Southern Africa. e) To summarise ectoparasite vector control and intervention strategies.

\section{Communicable Disease Epidemiology}

To understand the role of viral vector-borne diseases and in particular the main example of CCHF in Southern Africa, the following terms need to be clarified:

a) Vector: An arthropod that transmits an infectious agent mechanically or biologically.

b) Zoonosis: an infection or infectious disease transmissible under natural conditions from vertebrate animals to man that may manifest clinically or sub-clinically.

c) Arbovirus: viruses transmitted mainly by arthropod bites that are grouped as arthropod-borne viruses.

d) Host: a living creature human or animal that hosts an infectious agent under natural conditions. This is opposed to artificial or experimental conditions.

e) Reservoir: any human, animal, arthropod or natural substance in which an infectious agent can survive, replicate and be transmitted to a susceptible host.

f) Communicable disease: a disease transmitted by an infectious agent to a susceptible host. This can occur directly or indirectly through an intermediate host, plant or animal.

g) Vector control: any method to limit or eradicate mammals, birds, insects or arthropods which transmit disease pathogens.

h) CCHF: A clinical disease caused by a tick-borne zoonotic virus ranging from a non-specific febrile viral illness to severe disease with haemorrhagic manifestations resulting in high morbidity and mortality consequences. ${ }^{6,15,16}$

The key points to understanding the epidemiological approach to any communicable disease are highlighted by the following:

a) The infectious agent.

b) The mode of transmission.

c) The reservoir.

d) Possible intermediate host.

e) The main host.

f) Evolution of the infectious agent, where indicated. g) Environment, including climate and climate changes. ${ }^{1,6,17}$

As an example relevant to this review article, the infectious organism is the CCHF virus (CCHFV) which belongs to the family Bunyaviridae, genus Nairovirus. Arbovirus transmission occurs through the arthropod vector, the Ixodid tick Hyalomma $s p .^{18}$ The host is a human or another living animal that is susceptible to the infection or disease. The transmission cycle of the infectious agent takes place within an environment that will determine the success or severity of the disease and these factors can be diverse, including climate. ${ }^{1,6}$ The epidemiological approach to communicable disease assists in the design and maintenance of vector control and possible intervention strategies. ${ }^{6,19}$

\section{Arboviral diseases in southern Africa}

Many arthropod vectors can transmit viruses to human through bites, for example: mosquitoes, midges, ticks and flies, such as sand flies..$^{5,8,9}$ Table 1 lists selected arboviral diseases occurring in Southern Africa and also some of the common diseases seen in travellers returning home or visiting from other African countries. ${ }^{8}$ Table 1 has classified the diseases into routine screening diagnostic assay tests known as the viral haemorrhagic fever (VHF) screen and arbovirus screen, tested at the National Institute of Communicable Diseases in Johannesburg.

Table I Screening tests performed at the national institute of communicable diseases, Johannesburg, South Africa

\begin{tabular}{ll}
\hline VHF Screen & Arbovirus Screen \\
\hline RVF & RVF \\
Ebola & Sindbis \\
Marburg & Chikungunya \\
CCHF & West Nile \\
Lassa &
\end{tabular}

VHF:Viral Haemorrhagic Fever; RVF: Rift Valley Vever; CCHF: Crimean-Congo Haemorrhagic Fever

Rift Valley fever, a mosquito-borne arbovirus, has caused numerous epidemics and outbreaks in South Africa over time and periodic epidemics have been reported since $1951 .{ }^{20}$ Although there are periodic epidemics in Southern Africa, the most severe appears to have occurred during the autumn of 1975 after unusually heavy rains in the central regions of Southern Africa. Many humans were affected and seriously ill patients were hospitalized. ${ }^{20}$ Rift Valley fever has four clinical syndromes and can manifest with bleeding manifestations similar to CCHF. ${ }^{8}$ Travellers returning or coming to South Africa can be infected with other regional arboviral infections and/ or viral haemorrhagic fever viruses, as has been documented in the past and the literature (Rift Valley fever, Sindbis, Chikungunya and West Nile viruses are examples). ${ }^{21}$

\section{Crimean-Congo haemorrhagic fever - Virology and clinical presentation}

In 1967 a Soviet virologist registered a fatal case of Crimean haemorrhagic fever virus in the Catalogue of Arthropod-borne Viruses but four months earlier initial reports were published on another virus the authors named Congo virus. ${ }^{22,23}$ The International Committee on Taxonomy of Viruses proposed the name Congo-Crimean haemorrhagic virus but the Soviets insisted on CCHF and this was adopted as the official name in $1973 .{ }^{24}$ There are reports that could be missed in medical databases and epidemiological surveillance databases as there should also be searches for the "Congo-Crimean haemorrhagic fever virus" or the "Congo virus". 
The virus is a tick-borne zoonotic virus that is associated with a wide range of clinical signs and symptoms presenting with a nonspecific febrile illness to severe disease and fatal haemorrhaging. (Table 2) illustrates the full viral classification of CCHFV. ${ }^{14,25}$ This specific virus has a negative sense, single-stranded RNA genome which is circular and ambisense with three segments, namely large (L), middle (M) and small (S). A recent research study conducted in South Africa has revealed high frequency reassortment in the $\mathrm{M}$ segment and further studies are needed to relate this to a competitive advantage such as increased transmissibility and enhanced pathogenicity. ${ }^{14}$ When population genetics are considered, CCHFV is one of the most genetically diverse of the arboviruses and reassortment is widely described within its Bunyaviridae family. ${ }^{26}$ Reassortment amongst the genomic segments that occur during co-infection of ticks or vertebrates could have played a role in generating diversity in the $\mathrm{CCHFV}^{26}$

Table 2 Crimean-Congo haemorrhagic fever virus classification

\begin{tabular}{ll}
\hline Group & GroupV ((-)ss RNA) \\
\hline Order & Unassigned \\
Family & Bunyaviridae \\
Genus & Nairovirus \\
Species & Crimean-Congo haemorrhagic fever virus \\
\hline
\end{tabular}

((-) ssRNA: Negative Sense, Single Stranded RNA

The Hyalomma $s p$ tick is found throughout South Africa but mostly occurs in the drier areas. Various stages of the ticks feed on small animals and birds, while the adult ticks feed on larger animals such as sheep and cattle. ${ }^{9,15,27}$ When animals are slaughtered during the viraemic phase, the infection can be transmitted to humans and certain occupations may be susceptible or at an increased risk for infection with CCFHV. ${ }^{9,27}$ Transmission occurs by the following main mechanisms:

a) The bite of a viraemic tick.

b) Mechanical crushing of the viraemic tick with resultant exposure to the virus.

c) Contact with infected body parts and fluids of animals.

d) Contact with viraemic and infected humans or infected body fluids and excreta. ${ }^{27,28}$

Thus, clinicians need to elicit a thorough clinical history about possible exposure to CCHFV. ${ }^{12}$ After a short incubation period of about 3-6 days, typical flu-like symptoms appear. In a small percentage of patients (20-30\%) this may resolve after one week. However the majority of patients deteriorate clinically and haemorrhagic manifestations start to present within 3-5 days after the onset of the illness. ${ }^{29}$ Recovery can occur after 9-10 days from when the symptoms started but at least $30 \%$ of patients die within the second week after onset of the illness. ${ }^{29}$ Clinical pathology during the first five days of illness can be summarised as follows:

a) Leucopaenia or leucocytosis.

b) Thrombocytopenia.

c) Abnormal blood clotting profile (PI/PTT values).

d) Abnormal or raised liver enzymes. ${ }^{30}$

South African CCHF outbreaks have been described in the literature. One particular outbreak described at the Tygerberg Hospital involved the complicated screening of contacts, as at the same time there was an influenza epidemic. Both viral diseases have a similar early clinical presentation. Over four hundred listed contacts were identified and of these only 7 contracted the disease (1.5\%). Two of the 7 contacts had reportedly no direct contact with the index patient and this caused great concern about the dissemination of the virus. Full protective measures were applied. ${ }^{28}$

\section{Discussion}

\section{Ectoparasite vector control and intervention strategies}

There is no vaccine prophylaxis or effective therapeutic interventions (Ribavirin might be effective according to some in vitro studies ${ }^{9}$ ) currently available for CCHF. Where tick infestation is common, agricultural regulations should be strictly followed, such as de-ticking slaughter animals before transportation and delivery for slaughter. Work personnel should use personal tick avoidance measures such as the following:

a) Regular use of appropriate insect repellents;

b) Adequate protective clothing

c) Daily body inspection for adherent ticks, including the examination of the body regions particularly sought by ticks (example the groin regions). ${ }^{30}$

Ectoparasites (fleas, lice, bedbugs, mites and ticks) live on the outside of the body and there are various control measures to be considered, namely:

a) Personal hygiene.

b) Reduction of interpersonal crowding and the sharing of clothes.

c) Regular and effective washing of clothes and bedding.

d) Repellents.

e) Improved house construction (ectoparasites favour dark, dirty places).

f) Insecticides. ${ }^{8}$

Table 3 outlines the sequence of actions and intervention strategies to be followed with any communicable disease outbreak. ${ }^{6,28}$ International Health Regulations require that certain diseases be notified. Notification is a form of passive surveillance but active surveillance can be done by testing the tick viral vectors and infected animals. ${ }^{27}$ In South Africa, CCHF is a notifiable medical condition in terms of the National Health Act (Act 61 of 2003).

\section{Conclusion}

Understanding the epidemiology of arboviruses as communicable diseases gives an indication as to which vector control and intervention strategies to follow to combat the disease. Vector control remains the mainstay in the prevention of most arboviruses.

CCHF is the most important arboviral disease with haemorrhagic manifestations in South Africa. It is a tick-borne disease for which there is no vaccine prophylaxis or effective therapeutic interventions available at present.

CCHFV is a negative sense, single-stranded RNA virus with a three segmented genome that is classified in the Bunyaviridae family. Recent and numerous research studies have shown that members of the Bunyaviridae family are prone to genetic reassortment. It is likely that CCHFV genomes can give rise to possible genetic drift and shift in 
their arthropod vectors. Genetic analysis of Southern African CCHFV isolates confirms the high frequency of $\mathrm{M}$ segment reassortment and further studies are indicated to research the consequences of reassortment relating to CCHFV pathogenicity and transmissibility. Further research studies could improve intervention strategies for $\mathrm{CCHF}$ and other arboviral viruses and could enhance outbreak control measures and surveillance strategies.

Table 3 The sequence of actions to be followed with a communicable disease outbreak $^{6,28}$

- Identification of the infectious agent (diagnosis)

- Notification of the disease

- Treatment of the cases

- Interruption of transmission

- Prevention of recurrence

- Analysis of the outbreak and report/publication

- $\quad$ Surveillance

\section{Acknowledgements}

The author wishes to acknowledge the Zoonosis Research Unit and the Department of Medical Virology, University of Pretoria for the assistance in the preparation of this review article. The author wishes to thank and acknowledge Professor PGD Rautenbach, Department of Community Health, University of Limpopo, for assistance and advice in the preparation of this manuscript.

\section{Conflicts of interest}

None.

\section{References}

1. Gage KL, Burkot TR, Eisen RJ, et al. Climate and vectorborne diseases. Am J Prev Med. 2008;35(5):436-450.

2. Barclay E. Predicting the next pandemic. Lancet 2008;372(9643):1025-1026.

3. Taylor LH, Latham SM, Woolhouse ME. Risk factors for human disease emergence. Philos Trans R Soc Lond B Biol Sci.2001;356(1411):983-988.

4. Asokan GV, Ramanathan K Kasimanickam, Asokan V. Surveillance, response systems and evidence updates on emerging zoonoses: the role of one health. Infect Ecol Epidemiol. 2013;3(10).

5. Webber R. Chapter 16: Insect-borne diseases. In: Webber R (Eds.) Communicable diseases epidemiology and control $\left(2^{\text {nd }}\right.$ edn $), C A B$ International, Wallingford, Oxford, United Kingdom. 1997;pp.221-286.

6. Rautenbach PGD. Mosquito-borne viral infections in Southern Africa. CME: Your SA Journal of CPD: Medical virology. 2011;29(5):204-206.

7. Lara HH, Sepulveda-de Leon VH, Mureyko L, et al. Chikungunya in the United States. J Human Virol Retrovirol. 2014;1(3):00015.

8. Webber R. Chapter 17: Ectoparasite zoonosis. In: Communicable diseases, epidemiology and control ( $2^{\text {nd }}$ edn). CAB International, Wallingford, Oxford, United Kingdom. 1997;pp.287-308.

9. Prinsloo B. Arboviral diseases in Southern Africa. SA Fam Pract. 2006;48(8):25-28.

10. Bente DA, Forrester NL, Watts DM, et al. Crimean-Congo hemorrhagic fever: history, epidemiology, pathogenesis, clinical syndrome and genetic diversity. Antiviral Res. 2013;100(1):159-189.

11. Msimang V, Weyer J, Leman P, et al. Update: Crimean-Congo haemorrhagic fever in South Africa. Communicable Diseases Surveillance Bulletin. 2013;11(3):62-65.
12. MMWR Weekly. Congo-Crimean Hemorrhagic fever-Republic of South Africa. JAMA. 1984;252(18):2533, 2537.

13. Swanepoel R, Struthers JK, Shepherd AJ, et al. CrimeanCongo hemorrahagic fever in South Africa. Am J Trop Med Hyg. 1983;32(6):1407-1415.

14. Goedhals D, Bester PA, Paweska JT, et al. Next-generation sequencing of southern African Crimean-Congo haemorrhagic fever virus isolates reveals a high frequency of $\mathrm{M}$ segment reassortment. Epidemiol Infect. 2014;142(9):1952-1962.

15. Hoogstraal H. The epidemiology of tick-borne Crimean-Congo haemorrhagic fever in Asia, Europe and Africa. J Med Entomol. 1978;15(4):305-417.

16. Shepherd AJ, Swanepoel R, Cornel AJ, et al. Experimental studies on the replication and transmission of Crimean-Congo hemorrhagic fever virus in some African tick species. Am J Trop Med Hyg. 1989;40(3):326-331.

17. Anagnostou V, Papa A. Evolution of Crimean-Congo hemorrhagic fever virus. Infect Genet Evol. 2009;9(5):948-954.

18. Ergonul O. Crimean-Congo hemorrhagic fever virus: new outbreaks, new discoveries. Curr Opin Virol. 2012;2(2):215-220.

19. Marc Mertens, Katja Schmidt, Aykut Ozkul, et al. The impact of Crimean-Congo hemorrhagic fever virus on public health. Antiviral Research. 2013;98(2):248-260.

20. Van Velden DJ, Meyer JD, Olivier J, et al. Rift Valley fever affecting humans in South Africa: a clinicopathological study. S Afr Med J. 1977;51(24):867-871.

21. Briese T, Paweska JT, McMullan K, et al. Genetic detection and characterisation of Lujo virus, a new hemorrhagic fever-associated Arenavirus from Southern Africa. PLoS Pathog. 2009;5(5):1000455.

22. Chumakov MP, Butenko AM, Shalunova NV, et al. New data on the viral agent of Crimean hemorrhagic fever. Vopr Virusol. 1968;13(3):377.

23. Simpson DI, Knight EM, Courtois G, et al. Congo virus: a hitherto undescribed virus occurring in Africa. I. Human isolations - clinical notes. East Afr Med J. 1967;44(2):86-92.

24. Casals J. Antigenic similarity between the virus causing Crimean hemorrhagic fever and Congo virus. Proc Soc Exp Biol Med. 1969;131(1):233-236.

25. Elliott RM. Family Bunyaviridae. In: Regenmortel MHV et al. (Eds.), Seventh Report of the International Committee on Taxonomy of Viruses. ( $1^{\text {st }}$ edn), Academic Press, San Diego, USA, 2000;pp.1024.

26. Briese T, Calisher CH, Higgs S. Viruses of the family Bunyaviridae: are all available isolates reassortants? Virology. 2013;446(1-2):207-216.

27. Shepherd AJ, Swanepoel R, Shepherd SP, et al. Viraemic transmission of Crimean-Congo haemorrhagic fever virus to ticks. Epidemiol Infect. 1991;106(2):373-382.

28. Van Eeden PJ, Joubert JR, Van de Wal BW, et al. A nosocomial outbreak of Crimean-Congo haemorrhagic fever at Tygerberg Hospital. Part 1. Clinical features. S Afr Med J. 1995;68(10):711-717.

29. Bente DA, Forester NL, Watts DM, et al. Crimean-Congo hemorrhagic fever: history, epidemiology, pathogenesis, clinical syndrome and genetic diversity. Antiviral Res. 2013;100(1):159-189.

30. Swanepoel R, Gill DG, Shepherd AJ, et al. The clinical pathology of Crimean-Congo hemorrhagic fever. Rev Infect Dis. 1989;11(4):S794S800. 